Article

\title{
Experimental Measurement of Dolphin Thrust Generated during a Tail Stand Using DPIV
}

\author{
Frank E. Fish ${ }^{1, *(1)}$, Terrie M. Williams ${ }^{2}$, Erica Sherman ${ }^{3}$, Yae Eun Moon ${ }^{3}$, Vicki $\mathrm{Wu}^{3}$ and \\ Timothy Wei ${ }^{3}$ \\ 1 Department of Biology, West Chester University, West Chester, PA 19383, USA \\ 2 Center for Ocean Health, Long Marine Laboratory, University of California Santa Cruz, \\ Santa Cruz, CA 95060, USA; tmwillia@ucsc.edu \\ 3 Department of Mechanical and Materials Engineering, University of Nebraska, Lincoln, NE 68588, USA; \\ sherman.erica@gmail.com (E.S.); yaeeun.moon@gmail.com (Y.E.M.); vickiwu88@gmail.com (V.W.); \\ twei3@unl.edu (T.W.) \\ * Correspondence: ffish@wcupa.edu; Tel.: +1-610-436-2460
}

Received: 1 April 2018; Accepted: 14 May 2018; Published: 17 May 2018

\begin{abstract}
Estimation of force generated by dolphins has long been debated. The problem was that indirect estimates of force production for dolphins resulted in low values that could not be validated. Bubble digital particle image velocimetry (DPIV) measured hydrodynamic force production for swimming dolphins and demonstrated high force production. To validate the bubble DPIV and reconcile force production measurements, two bottlenose dolphins (Tursiops truncatus) performing tail stands were measured with bubble DPIV. Microbubbles were generated from a finely porous hose and compressed air source. Displacement of the bubbles by the propulsive motions of the dolphin was tracked with a high-speed video camera. Oscillations of the dolphin flukes generated strong vortices and a downward directed jet flow into the wake. Application of the Kutta-Joukowski theorem measuring vortex circulations yielded forces up to $997.3 \mathrm{~N}$. Another video camera recorded body height above the water surface to determine the mass-force of the dolphin above the water surface. For the dolphin to hold its position above the water surface, the mass-force approximately balanced the vertical hydrodynamic force from the flukes. The results demonstrated the fluke motions generate high sustained forces roughly equal to the dolphin's weight out of the water. Bubble DPIV validated high forces measured previously for thrust generated in swimming by animals and demonstrated a more accurate technique compared to standard aerodynamic analysis.
\end{abstract}

Keywords: bubble DPIV; tail stand; Tursiops; thrust; microbubbles

\section{Introduction}

Swimming by animals requires the transfer of momentum from the organism to the water. The rate of change of momentum generates the propulsive thrust that is indistinguishable from the resistive drag on the actively swimming body [1]. The momentum imparted to the water by a propulsor is visualized in a thrust-type (reverse von Kármán street) wake with a central momentum jet, which is directed through the center of a staggered array of vortex rings [2-7]. This exchange of momentum and its measurement have been at the core of examinations of forces and energetics of swimming by animals [8]. To this end, various studies have sought to visualize the wake [9-15]. The movement of fluid within the wake represents the physical manifestation of the momentum transfer.

Digital particle image velocimetry (DPIV) has been used as an effective technique to quantify the wake and determine hydrodynamic forces. DPIV has been used to examine flow patterns associated with feeding currents and to measure the thrust and lift forces associated with the propulsion of aquatic 
animals, including fish, squid, and crustaceans [9-15]. Typically, in using DPIV, microscopic reflective particles are illuminated with a laser sheet. The deflections of the particles in the plane of the laser sheet are recorded with a high-speed digital video camera. Sequential images of the particles within given areas are cross-correlated to determine the velocity field and vorticity, which can be used to compute the forces generated about an organism. A variant of standard DPIV was used to measure the hydrodynamic force production for dolphins during free swimming [7]. As dolphins could not be exposed experimentally to lasers and reflective particles, animals swam along a sheet of microbubbles in natural light (i.e., bubble DPIV). The results of the experiment demonstrated high force production for actively swimming dolphins.

DPIV has been used widely by engineers on static systems that can be independently validated with experimental data from standardized rigid shapes [16]. However, similar validations have not been applied to DPIV results on actively swimming animals. The problem is that there is no proper control or reference value that can be compared with the drag or thrust values of actively swimming animals. The drag is higher for an actively swimming animal compared to a static, rigid body or reference flat plate $[17,18]$. The dynamic self-propulsion of swimming animals modifies the time-dependent flow compared to that of a rigid body [1]. The problem in estimating the thrust produced by self-propelled animals is that the motions of the body produce drag and thrust simultaneously, which cannot be separated and thus momentum losses and gains are co-mingled [1]. Unlike analysis of a ship traveling at a constant velocity, the drag on the rigid ship's hull can be separated from the thrust produced by the propeller. For the ship, the propulsive and resistive forces sum to zero.

To validate previous results of DPIV studies on live animals and reconcile force production measurements, bottlenose dolphins performing tail stands were measured with bubble DPIV. Tail stands are defined as a behavior in which a vertically oriented dolphin lifts half or more of its body out of the water. It was expected that the thrust determined from bubble DPIV would match the weight of the dolphin supported above the water surface. For the dolphin to hold its position above the water surface, the mass-force would approximately balance the vertical hydrodynamic force generated by the oscillating flukes. The results of the bubble DPIV were also compared to measurements calculated from lifting wing theory.

\section{Materials and Methods}

\subsection{Experimental Animals and Microbubbles}

Experiments were conducted on two adult male bottlenose dolphins (Tursiops truncatus Montagu) (Table 1), which were maintained at the Long Marine Laboratory at the University of California Santa Cruz. The animals were measured for their body mass, length, fluke span, fluke chord, and fluke area. Measurements of the flukes were determined from scaled planar photographs of the flukes. The fluke chord was measured at the base of the flukes as the linear distance from leading to trailing edge. The dolphins were held in a large, outdoor, marine pool $(15.2 \mathrm{~m} \times 9.1 \mathrm{~m} \times 4.0 \mathrm{~m})$. There were four underwater viewing windows $(1.4 \mathrm{~m} \times 1.2 \mathrm{~m})$ along one side of the pool.

Table 1. Morphometrics of dolphins (Tursiops truncatus) tested.

\begin{tabular}{cccccc}
\hline Dolphin & Length $(\mathbf{m})$ & Mass $(\mathbf{k g})$ & Fluke Span $(\mathbf{m})$ & Fluke Chord $(\mathbf{m})$ & Fluke Area $\left(\mathbf{m}^{\mathbf{2}}\right)$ \\
\hline Primo & 2.41 & 181.5 & 0.67 & 0.21 & 0.094 \\
Puka & 2.46 & 205.5 & 0.68 & 0.23 & 0.110 \\
\hline
\end{tabular}

Dolphins were trained to perform a tail stand within a sheet of microbubbles. As previously explained [7], microbubbles were generated from a compressed air source (i.e., SCUBA tank) that was forced into a finely porous hose. The hose was located at the bottom of the pool, about $1 \mathrm{~m}$ from the pool wall. The hose was held within a metal guide, which weighted the hose and kept it straight. 
The microbubbles were initially less than $1 \mathrm{~mm}$ in diameter, though there was some coalescence as they rose to the surface. The bubble sheet was oriented parallel to an underwater viewing window. The bubble sheet extended from the bottom of the pool to the surface with a length of $6.2 \mathrm{~m}$ and a thickness of $2 \mathrm{~cm}$.

Under trainer control, each dolphin executed a series of tail stands. The dolphin aligned itself in the bubble sheet so that its dorsoventral tail motions remained in line with the sheet. The height of the tail stand was controlled by the trainer. The dolphin would raise itself up to a float target at the end of a pole that was held above the dolphin's rostral tip (Figure 1). The height out of the water was at or above the position of the center of mass (CM), which was located at the anterior insertion of the dorsal fin, about $41 \%$ of body length [18]. The dolphin held its position above the surface of the water without a noticeable change in height for a period of $2 \mathrm{~s}$. The height above the water was measured using the eye as a marker, as the tip of the rostrum was not always oriented vertically.

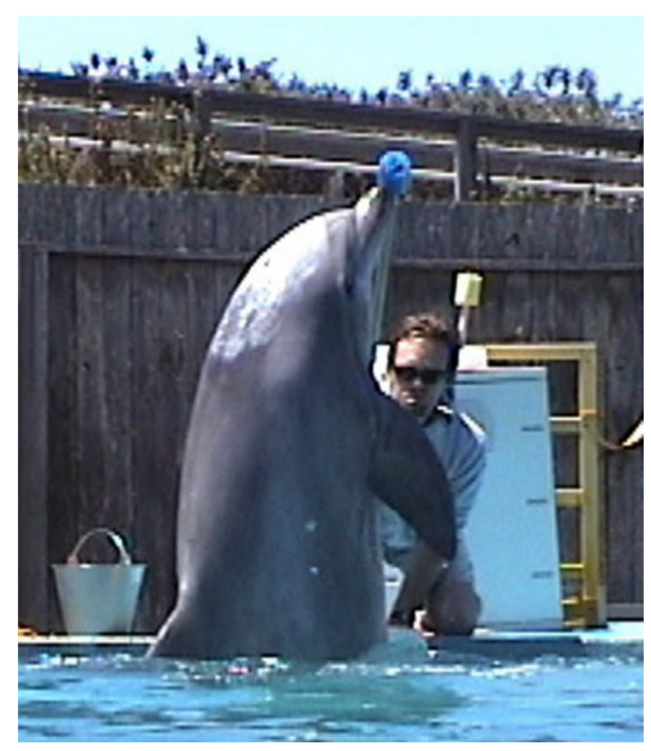

Figure 1. Dolphin performing tail stand. The dolphin was trained to target a blue float and rise to a height out of the water under trainer control.

\subsection{Bubble DPIV}

The size and density of the microbubble sheet provided sufficient resolution for DPIV relative to the dimensions and speed of movements of the dolphins $[7,19,20]$. A Redlake MotionPro X3 high-speed video camera recorded the movements of the microbubbles through the underwater viewing window as the microbubbles were displaced by strokes of the dolphin's flukes. The camera had nominally $1000 \times 1000$ pixel resolution and could frame at rates up to 1000 frames per second at full resolution. The $2 \mathrm{D}$ velocity fields, due to the displacements of the microbubbles, were computed with DPIV software. This is a technique in which tracer images-microbubbles in this case-are tracked from one video frame to the next using standard cross-correlation techniques applied to an array of small, square interrogation windows. In-house software was used in this study and a previous study [7] as described by Hsu and Hsu et al. [21,22].

The microbubbles generated in this study were slightly positively buoyant. This resulted in a small, uniform upward velocity component in the flow measurements due to the bubble rise velocity. This was essentially a constant "dc" velocity superimposed on the true flow velocity. By taking data without the dolphins present, this mean rise velocity was measured and subtracted from the dolphin swimming measurements. Because the rise velocity was uniform and constant across the entire field of view, there was no artificial circulation (see following paragraph) added to the measurements. 
Using the underwater, high-speed video, the stroke cycle frequency $(f, \mathrm{~Hz})$ and the amplitude of heave $(A, \mathrm{~m})$ were measured. The frequency was the inverse of the period of a stroke cycle. The amplitude of heave was the horizontal distance traversed by the trailing edge of the flukes for one half-stroke cycle. For comparison between the two test dolphins and with data from other studies, a length-specific amplitude was calculated as $A / L$, where $L$ is the body length of the dolphin.

\subsection{Thrust Calculations}

For the tail stand, the motion of the flukes is similar to an oscillating finite wing canted at an angle of attack. The flukes generate lift that is in-line to the longitudinal axis of the body. The anterior body of the dolphin is held out of the water by the lift force, which equals the weight not supported by buoyancy.

Oscillations of hydrofoils like the dolphin's flukes produced pairs of counter-rotating vortices in a reverse Karman vortex street, as determined by the direction of motion of the flukes [3]. By defining and integrating around a closed contour around the core of each vortex, the circulation, $\Gamma$, which is proportional to the lift, was calculated:

$$
\Gamma=\int V \bullet d s,
$$

where $V$ are the velocities around the contour and $d s$ are the corresponding differential tangent vectors on the contour. Circulation at a vector location was calculated by summing the calculated vorticity, $\partial u / \partial y-\partial v / \partial x$, at that location multiplied by the differential area of the grid element; this is a standard application of Stoke's theorem covered in an introductory fluid mechanics text. The total circulation of a vortex was calculated by summing the individual circulations over a rectangular region containing the vortex. The height and width of the grid were varied to ensure that the largest circulation value was found; increasing or decreasing the area would result in a lower circulation value. For ease of calculation, a square contour aligned with the coordinate directions was chosen to simplify the integration. The size of the contour was chosen to be small enough to capture the vortex core-that is, excluding the outer region of the vortex where diffusion and dissipation of vorticity would yield a reduced vortex strength-yet large enough to ensure accurate integration. A sensitivity analysis of size and placement of the contour is provided in Legac [23].

The average $V_{\text {tail }}$ was determined by measuring the maximum distance traveled by the flukes during each half stroke multiplied by the number of half strokes in each direction divided by the total time for those strokes. The $V_{\max }$ was the maximum speed of the flukes moving across the axis of the body in $0.5-0.10 \mathrm{~s}$. For these measurements, a non-zero value of $\Gamma$ implied the existence of a thrust-producing vortex.

Thrust production was determined from the vortex strength for small-amplitude swimming [7]. Making the first-order approximation that the flukes were simple two-dimensional hydrofoils, the Kutta-Joukowski theorem related $\Gamma$ and thrust, $T$, produced by the dolphin:

$$
T=\rho \Gamma V_{t a i l} b,
$$

where $V_{\text {tail }}$ is the dorsoventral velocity of the tail, perpendicular to the longitudinal axis of the body, $\rho$ is the fluid density (1024 $\mathrm{kg} \cdot \mathrm{m}^{-3}$ for seawater), and $b$ is the span of the dolphin's flukes. To avoid errors in momentum estimation due to the use of PIV, analysis was performed on sequences in which the vortex ring cross-sections were circular [24].

Instantaneous thrust was computed using circulation and $V_{\max }$. This is the thrust generated for one stroke. The average thrust was computed using circulation and average $V_{\text {tail }}$. This is better thought of as the instantaneous thrust scaled by the fraction of time the dolphin's tail is acting as a hydrofoil.

$T$ was also computed using aerodynamic theory according the equation:

$$
T=0.5 \rho A C_{L} V_{\text {tail }^{2}}
$$


where $A$ is the planar surface area of the flukes and $C_{L}$ is the lift coefficient. $C_{L}$ was based on the cross-sectional geometry of the flukes of a $T$. truncatus with respect to the angle of attack and was obtained from Fish et al. [25]. It should be noted here that the term lift coefficient, $C_{L}$, in this context is actually a thrust coefficient. As discussed in Fish et al. [7], the "lift" generated by the lateral motion of the tail is along the axis of the dolphin's body (i.e., in the thrust direction). Because $C_{L}$ is the commonly accepted designation for wings, it is used here. The angle of attack was measured from video images taken during mid-stroke as the angle between the fluke chord and the horizontal.

A Sony Digital 8 Handycam (HDR SR11; 10.2 megapixel resolution, Sony, Tokyo, Japan) camera was used to collect video on the height above the water attained by the dolphin during a tail stand. The camera was positioned on the far side of the pool, approximately $9 \mathrm{~m}$ from the dolphin.

To maintain vertical position during a tail stand (Figure 2), all the forces of the system must sum to zero as:

$$
T+F_{b}-W=0,
$$

where $F_{b}$ is the buoyant upward force and $W$ is the weight of the dolphin. In this configuration, the thrust, $T$, generated by the tail movements balances the proportion of the dolphin's weight that is above the water surface and thus the weight minus the buoyant force, $F_{b}$ :

$$
T=W-F_{b} .
$$

$F_{b}$ is equal to the mass of water displaced by the submerged portion of the body $\left(m_{\text {sub }}\right)$ times the acceleration due to gravity $\left(g ; 9.8 \mathrm{~m} \cdot \mathrm{s}^{-2}\right)$ :

$$
F_{b}=m_{\text {sub }} \cdot g \text {. }
$$

The proportion of the dolphin's weight that is above the surface of the water, and therefore the thrust, required to maintain height is:

$$
T=h / L \cdot W,
$$

in which $h$ is the height of the rostral tip above the surface of the water (Figure 2). As $h$ increases, $T$ will increase linearly and $F_{b}$ will decrease (Figure 2). When fully submerged $(h<0), F_{b}$ will approximate the weight of the dolphin, whereas if dolphin can hold its body out of the water $h \approx L$, the thrust from the flukes will nearly equal the weight of the animal.

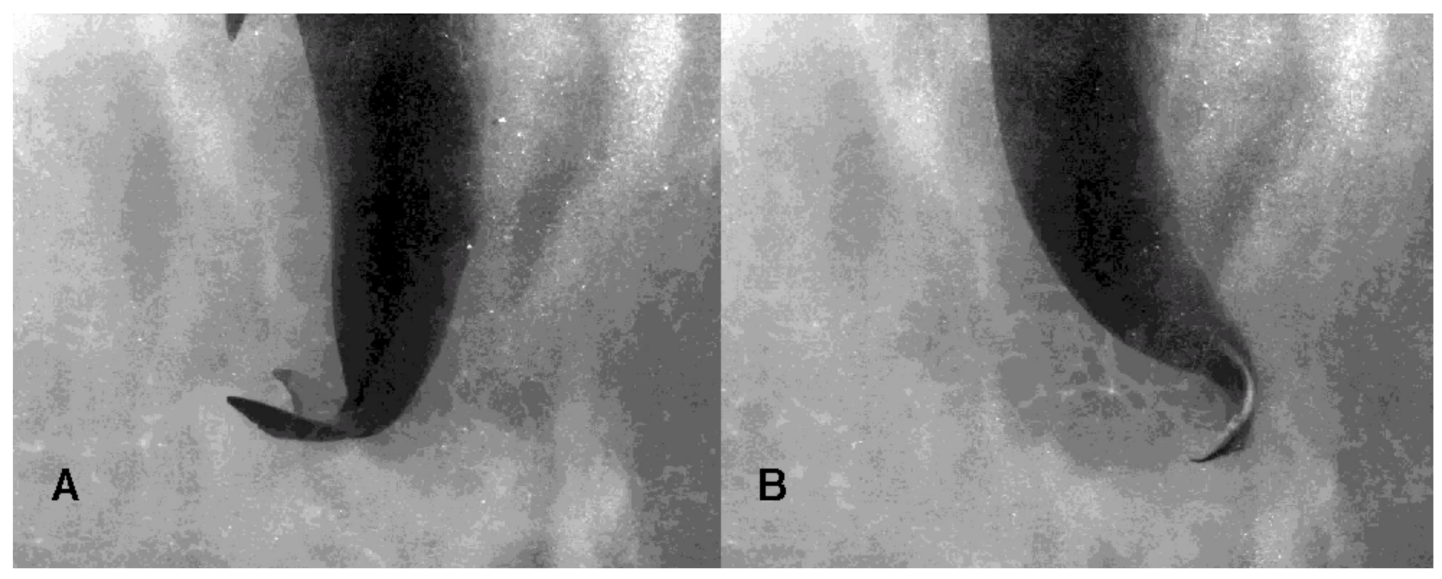

Figure 2. Spanwise bending of the flukes in mid-stroke (A) and chordwise bending of the flukes at the end of the stroke $(\mathbf{B})$. 


\subsection{Statistical Analysis}

Variation about means was expressed as \pm one standard deviation (SD). All least-squares regressions and correlation coefficients, $r$, were calculated using KaleidaGraph software (version 4.03, Synergy Software, Reading, PA, USA). Correlation coefficients were determined to be statistically significant at a level of $p<0.05$.

\section{Results}

\subsection{Tail Stand}

A total of 51 separate trials were made by the two dolphins (28 and 23 trials for Primo and Puka, respectively), with morphometrics of the two dolphins are provided in Table 1. Relative to the vertical position of the eye above the surface of the water, the dolphins performed tail stands with the position of the eye at $0.74 \pm 0.16 \mathrm{~m}$ above the surface of the water. The mean proportion of body mass of the dolphins supported above the water during the tail stands was $0.5 \pm 0.1$, which is equivalent to a weight of $906.7 \pm 214.8 \mathrm{~N}$. For the six trials that were examined with bubble DPIV and aerodynamic analysis, the mean weight supported above the surface of the water was $882.2 \pm 165.7 \mathrm{~N}$.

The majority of trials were discarded because the dolphin did not center itself on the bubble sheet, maintain a vertical position, or have the fluke stroke perpendicular to the camera. Only six trials were considered acceptable. In these trials, the flukes oscillated dorsoventrally in a sculling motion. On the downstroke, the ventral side of the flukes faced downward and was canted at a slight positive angle to the horizontal, whereas on the upstroke, the flukes were flipped so that the dorsal side of the flukes faced downward and was canted with a slight positive angle to the horizontal. Considerable spanwise bending of the flukes was observed as the flukes moved through the stroke across the axis of the body, whereas chordwise bending was noted at the transitions in direction change (Figure 2). The mean $f$ was $2.70 \pm 0.12 \mathrm{~Hz}$. The mean $A$ was $0.82 \pm 0.04 \mathrm{~m}$, which gave an $A / L$ of $0.34 \pm 0.02$.

\subsection{Bubble DPIV}

The oscillatory motions of the flukes produced pairs of counter-rotating vortices with a jet flow that were ejected downward into the wake throughout each propulsive cycle. One vortex was generated at the end of each half stroke as the flukes reversed direction. The vortex was shed from the trailing edge of the flukes (Figure 3).

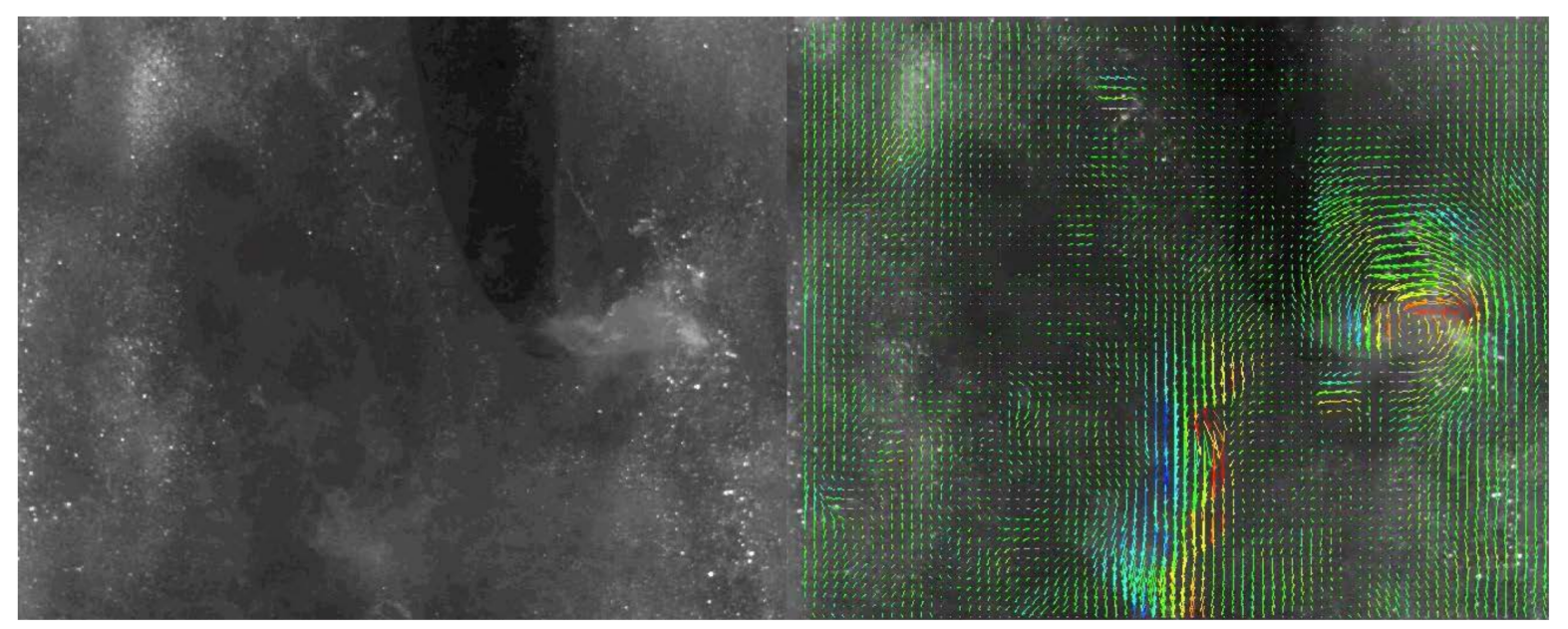

(A)

(B)

Figure 3. Displacement of bubbles (A) and vector flow from digital particle image velocimetry (DPIV) (B) as the flukes are moving to the left. The colored vectors show the vortex and jet flow. 
The mean values for the average $\left(V_{\text {tail }}\right)$ and maximum $\left(V_{\max }\right)$ velocities of the flukes were $3.03 \pm 0.47 \mathrm{~m} \cdot \mathrm{s}^{-1}$ and $4.16 \pm 0.52 \mathrm{~m} \cdot \mathrm{s}^{-1}$, respectively. The mean circulation in the vortices generated by the flukes was $0.370 \pm 0.136 \mathrm{~m}^{2} \cdot \mathrm{s}^{-1}$, as computed from Equation (1).

Application of the Kutta-Joukowski theorem on measured vortex circulations yielded average thrust varying from 560.0 to $997.3 \mathrm{~N}$ with a mean value of $739.7 \pm 137.8 \mathrm{~N}$. The percent difference between the computed thrust of the individual trails compared to the weight supported out of the water varied from $1.3 \%$ to $36.0 \%$ with an average of $16 \%$. In two cases, the difference between the weight supported out of the water and thrust estimated varied only by $1.3 \%$ and $1.8 \%$.

The calculated thrust increased linearly with increasing weight supported above the water surface (Figure 4) according to the regression equation:

$$
T=242.94+0.56 W
$$

The $r$ value for the regression equation was 0.62 .

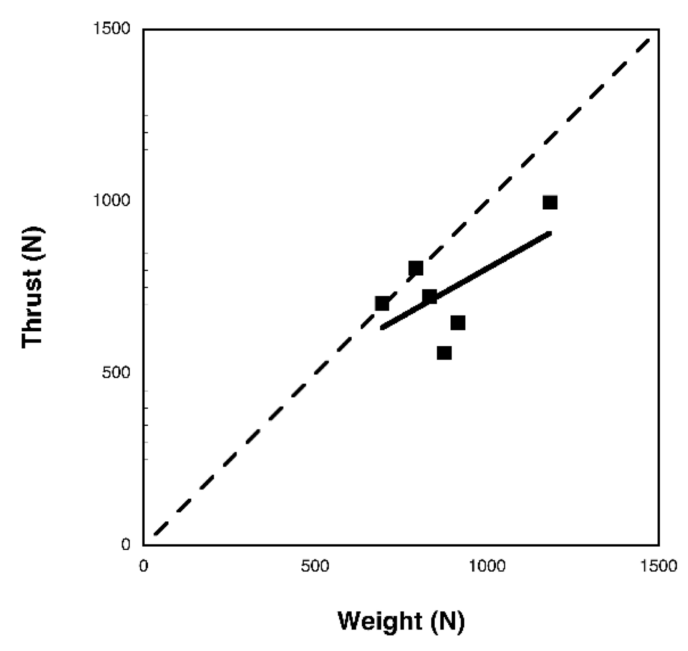

Figure 4. Plot of thrust measured using bubble DPIV with respect to weight supported out of the water. The solid line represents the regression of the data collected. The dashed line is line of equality for thrust produced to support the weight of the dolphin above the surface of the water during a tail stand.

The average thrust-to-weight ratio was 0.4 . The range of thrust values corresponded to mechanical power outputs of 1555.0-2616.5 W. As the percentage of adult body mass comprising the locomotor muscles of dolphins was found to be $25.7 \%$ [26], the maximum mass-specific muscle power output then was $27.5-52.4 \mathrm{~W} \cdot \mathrm{kg}^{-1}$. These values were within the range of power outputs for vertebrate muscle $[27,28]$ and were consistent with the values measured by bubble DPIV for freely swimming dolphins [7].

\subsection{Aerodynamic Analysis}

As the flukes were swept through the stroke cycle, the angle of attack varied from $12.0^{\circ}$ to $17.0^{\circ}$ with a mean of $14.7 \pm 1.8^{\circ}$. Such high angles of attack without stall are possible for oscillating foils until the angle exceeds $30^{\circ}$ [29]. Using $V_{\text {tail }}$ with Equation (3), the average thrust varied from 582.3 to $1306.6 \mathrm{~N}$ with a mean value of $892.1 \pm 283.8 \mathrm{~N}$. The thrust based on aerodynamic analysis was $20.6 \%$ higher than the average thrust computed from bubble DPIV (Figure 5). Comparisons of the thrust calculated from the aerodynamic analysis for individual trials differed on average from the associated weight supported during tail stands by $32.4 \%$ (Figure 5 ). 


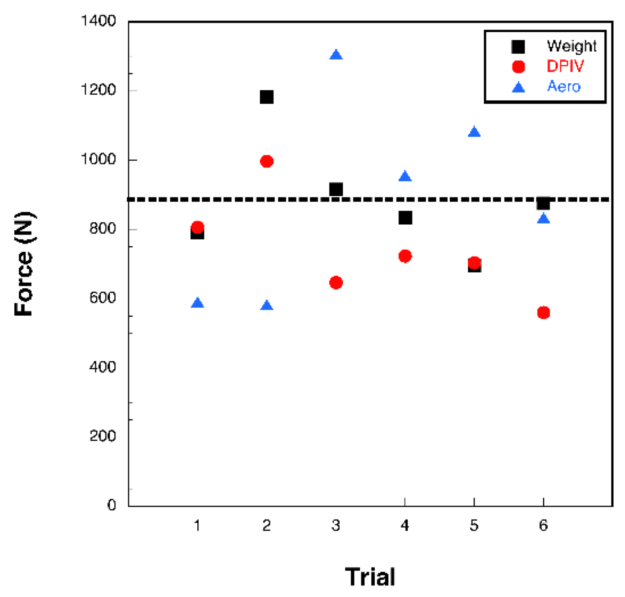

Figure 5. Comparison of the methods of bubble DPIV and aerodynamic analysis for each experimental trial. Symbols represent the weight supported out of the water during a tail stand (black squares), thrust measured using bubble DPIV (red circles), and thrust measured using aerodynamic analysis (blue triangles). The dashed line is the mean weight supported during a tail stand for the six experimental trials.

\section{Discussion}

Cetaceans (whales, dolphins, porpoises) are extremely energetic, powerful, and fast swimmers $[7,30,31]$. The mechanics of swimming by cetaceans entails propulsive lift-based oscillatory movements of the caudal flukes $[7,32,33]$. The flukes are lateral extensions from the tail of cetaceans with a wing-like planform and symmetrical cross-sectional profile. Estimation of force generated by the motion of the flukes for swimming dolphins has long been debated. The problem has been that early indirect estimates of force production for dolphins resulted in low values that could not be validated $[7,34,35]$.

A simple computational approach led to the erroneous "Gray's Paradox" [34,35]. The paradox resulted from a miscalculation in which the drag power (i.e., thrust power) for a dolphin to swim at high speed was considered to be higher than the maximum power output from the available propulsive muscle mass [34]. The only resolution to the paradox was to consider the boundary layer to be laminar, producing a low frictional drag. This conclusion was asserted despite a high Reynolds number for the dolphin indicating turbulent flow. Review of the assertions that were the basis for Gray's Paradox demonstrated that high muscle power output and high thrust power were possible for swimming dolphins [35].

Early experiments undertaken by Rosen [9] to visualize the flow around a dolphin included photographs of vortices in the spray of a leaping dolphin, attaching a dye patch on the head, having the dolphin swim through a two-dimensional screen of negatively buoyant plastic particles, and having the dolphin swim along a screen of bubbles. The latter experiment did not provide satisfactory results, as the bubble were too buoyant due to their size $(\leq 3.0 \mathrm{~mm})$. The rate of rise by the bubbles $(0.3-0.5 \mathrm{~m} / \mathrm{s})$ could not be subtracted at the time from the flow induced by the dolphin.

The use of the direct method of bubble DPIV has inherent interest as a technique to resolve controversies regarding the swimming performance of dolphins. A study by Fish et al. [7] using bubble DPIV indicated high thrust production for free-swimming dolphins. However, the technique had not been fully validated in a controlled experiment. Examination of the tail stand with bubble DPIV provided a unique set of circumstances to measure the effectiveness of the technique. For the tail stand, the oscillatory motion of the flukes is analogous to a finite wing moving back and forth horizontally in the water at an angle of attack. With each transit, the flukes (or wing) generates lift. The motion of the flukes is perpendicular to the axis of the body, and the "lift" vector (thrust) generated by the flukes 
therefore points along the body axis toward the head. This lift force, in turn, holds the dolphin up out of the water.

On average, bubble DPIV slightly underestimated the thrust necessary to support the weight of a dolphin performing a tail stand. However, some estimates of thrust corresponded tightly with the weight of the dolphin above the waterline. In comparison to estimates of thrust production based on the aerodynamic analysis, the bubble DPIV demonstrated greater accuracy. However, potential observational errors may have occurred to limit the accuracy of the technique. The cameras were not synchronized. Therefore, the exact video frames for measurement of thrust and the height of the dolphin above the surface of the water may not have exactly coincided. However, the data for both thrust and height were obtained when the animal had reached its maximum height. In addition, measurement of the exact height of the dolphin may have been affected by the motions of the dolphin in producing small waves, which would limit an exact measurement of the distance between the eye and the surface of the water. Another potential error was in the estimation of the center of mass of the specific dolphins examined in this study. The center of mass was determined from published results as a proportion of body length [18]. Lastly, the dolphins had to maintain an exact position and orientation in the bubble curtain to capture the vorticity for thrust calculations. This error was limited by choosing only those trials in which the dolphin maintained the proper position above the bubble curtain. This requirement reduced the number of suitable tests for examination to approximately $12 \%$ of all trials. The orientation within the bubble curtain was important in limiting three-dimensional effects. Such three-dimensional considerations may be dealt with in future tests with the application of volumetric DPIV as used to study swimming by fish and squid [36-38].

The use of thrust estimates from the aerodynamic analysis has more severe potential errors. The aerodynamic analysis was quasi-steady. The angle of attack of the flukes was determined from still images as the flukes crossed the longitudinal axis. Through the stroke cycle, the angle of attack would change particularly at transitions from upstroke to downstroke and vice versa. Such changes would affect the lift coefficient. The lift coefficient itself was based on a flat, rigid fluke with a symmetrical foil section. Throughout the stroke, the flukes demonstrated flexibility. Cambering by the flukes [25] and changes in the projected planar area were not incorporated into the aerodynamic calculations. As the bubble DPIV only examines the wake and magnitude of the circulation, changes in angle of attack, cambering, and planar area are already incorporated in the calculations of thrust.

The measurement of $f$ was in the range of values obtained from free-swimming dolphins. Using the regression equation from Fish [33], $f$ for the tail stand was equivalent to a bottlenose dolphin swimming at $5.76 \mathrm{~m} / \mathrm{s}$, which is within the range of swimming speeds for dolphins [31]. However, $A$ and $A / L$ were larger than values for steady-swimming bottlenose dolphins [7,33]. Dolphins swimming steadily typically have an $A / L$ of $0.15-0.2$, whereas the $A / L$ for the tail stand was $70-127 \%$ larger. However, $A / L$ of the tail stand was within the range of $0.20-0.50$ for large-amplitude strokes [39]. These large-amplitude strokes are associated with accelerations at the start of horizontal swims and at the beginning of the descent and ascent phases of dives. It is evident that the kinematics of the tail stand are similar to the stroke used for accelerations as an unsteady form of locomotion.

A surprising result of the examination of hydrodynamic tail stand of dolphins is the similarity with the hovering flight of hummingbirds. In both cases, the propulsive movements of both the wings and flukes direct a downward jet flow and associated vorticity. In both cases, the downward momentum developed from the kinematics of the propulsor must be given to the fluid at a rate equal to the supported weight of the animal [40]. In hovering flight, with the body oriented vertically in a head-up position, the hummingbird beats its wings horizontally [41,42]. In the downstroke, the wings move through the air at a positive angle of attack with the dorsum of the wing oriented upward. On the upstroke, the wing rotates along its length at the wrist so that the ventral surface is directed upward as it is reciprocated in the opposite horizontal direction with a positive or near-zero angle of attack [41-43]. These movements were similar to the actions of the dolphin flukes, which were flipped as the flukes were transitioned between the up- and downstrokes. 
Despite the similarity of tail stands in water by dolphins and hovering in air by hummingbirds, flow visualization indicates differences in the mechanics of support within the respective fluids. The vorticity shed by the oscillations of the flukes is generated as a reverse Kármán (thrust-type) vortex street [3]. The vortex street is produced as bound vortices are shed from the oscillating flukes at each half stroke as the direction of the flukes is reversed. The vortices are convected downward producing a staggered array of vortices with opposite spin with a downward jet flow centered within the vortex street. This pattern represents a symmetrical generation of thrust (i.e., lift) between the upstroke and downstroke. On the other hand, the thrust (i.e., lift) for hovering hummingbirds is asymmetrically generated. The stopping vortex at the end of the upstroke and starting vortex at the beginning of the downstroke merge into one [44]. The downstroke was determined to be stronger than the upstroke and to support $66-75 \%$ of the weight of the bird $[45,46]$. This asymmetry produced a pulsed vortex wake that is composed of a single merged vortex ring or bilateral vortex loop [44-46]. The difference in the wake structure between hovering hummingbirds and tail stands in dolphins reflects differences in the forces being developed by each species [47].

\section{Conclusions}

The results of bubble DPIV demonstrated that the fluke motions generated high levels of sustained force, which was roughly equal to the dolphin's weight supported above the surface of the water during a tail stand. Bubble DPIV, therefore, validated high forces measured previously from bubble PIV for thrust generated in free swimming. This technique can be used in aquarium settings and potentially in the field to visualize flow fields and calculate thrust production for large swimming animals. Furthermore, the physics of high-energy maneuvers, such as the tail stand, has engineering application for understanding ballistic performance across the air/water interface.

Author Contributions: The primary contribution for writing the article was performed by F.E.F. Analysis of data were performed by F.E.F., E.S., Y.E.M., V.W., and T.W., T.M.W. made available and maintained the dolphins for filming at the Long Marine Laboratory of the University of California Santa Cruz.

Funding: The work was supported by a grant from the Multidisciplinary University Research Initiative of the Office of Naval Research [N000141410533, 2014]. No funds were received for covering the costs to publish in open access.

Acknowledgments: We wish to express our appreciation to Traci Kendall, Beau Ritter and the training staff of the Long Marine Laboratory of the University of California Santa Cruz. We particularly thank the work of our dolphin assistants, Primo and Puka. Anthony Nicastro is acknowledged for his advice in analysis of the results.

Conflicts of Interest: The authors declare no conflict of interest.

\section{References}

1. Schultz, W.; Webb, P. Power requirements of swimming: Do new methods resolve old questions? Integr. Comp. Biol. 2002, 42, 1018-1025. [CrossRef] [PubMed]

2. Wu, T.Y. Hydrodynamics of swimming propulsion. Part 1 . Swimming of a two-dimensional flexible plate at variable forward speeds in an inviscid fluid. J. Fluid Mech. 1971, 46, 337-355. [CrossRef]

3. Weihs, D. Semi-infinite vortex trails, and their relation to oscillating airfoils. J. Fluid Mech. 1972, 54, 679-690. [CrossRef]

4. Triantafyllou, G.S.; Triantafyllou, M.S.; Grosenbaugh, M.A. Optimal thrust development in oscillating foils with application to fish propulsion. J. Fluids Struct. 1993, 7, 205-224. [CrossRef]

5. Anderson, J.M.; Streitlien, K.; Barrett, D.S.; Triantafyllou, M.S. Oscillating foils of high propulsive efficiency. J. Fluid Mech. 1998, 360, 41-72. [CrossRef]

6. Fish, F.E.; Lauder, G.V. Passive and active flow control by swimming fishes and mammals. Ann. Rev. Fluid Mech. 2006, 38, 193-224. [CrossRef]

7. Fish, F.E.; Legac, P.; Williams, T.M.; Wei, T. Measurement of hydrodynamic force generation by swimming dolphins using bubble DPIV. J. Exp. Biol. 2014, 217, 252-260. [CrossRef] [PubMed]

8. Webb, P.W. Hydrodynamics and energetics of fish propulsion. Bull. Fish. Res. Board Can. 1975, 190, 1-158.

9. Rosen, M.W. Experiments with swimming fish and dolphins. Am. Soc. Mech. Eng. 1961, 61-WA-203, 1-11. 
10. Drucker, E.G.; Lauder, G.V. Locomotor forces on a swimming fish: Three-dimensional vortex wake dynamics quantified using digital particle image velocimetry. J. Exp. Biol. 1999, 203, 2393-2412.

11. Drucker, E.G.; Lauder, G.V. Experimental hydrodynamics of fish locomotion: Functional insights from wake visualization. Integr. Comp. Biol. 2002, 42, 243-257. [CrossRef] [PubMed]

12. Videler, J.J.; Muller, U.K.; Stamhuis, E.J. Aquatic vertebrate locomotion: Wakes from body waves. J. Exp. Biol. 1999, 202, 3423-3430. [PubMed]

13. Videler, J.J.; Stamhuis, E.J.; Müller, U.K.; van Duren, L.A. The scaling and structure of aquatic animal wakes. Integr. Comp. Biol. 2002, 42, 988-996. [CrossRef] [PubMed]

14. Bartol, I.K.; Krueger, P.S.; Thompson, J.T.; Stewart, W.J. Swimming dynamics and propulsive efficiency of squids throughout ontogeny. Integr. Comp. Biol. 2008, 48, 720-733. [CrossRef] [PubMed]

15. Van Duren, L.A.; Stamhuis, E.J.; Videler, J.J. Copepod feeding currents: Flow patterns, filtration rates and energetics. J. Exp. Biol. 2003, 206, 255-267. [CrossRef] [PubMed]

16. Abbott, I.H.; von Doenhoff, A.E. Theory of Wing Sections; Dover: New York, NY, USA, 1959.

17. Lighthill, J. Mathematical Biofluiddynamics; Society for Industrial and Applied Mathematics: Philadelphia, PA, USA, 1975.

18. Fish, F.E. Balancing requirements for stability and maneuverability in cetaceans. Integr. Comp. Biol. 2002, 42, 85-93. [CrossRef] [PubMed]

19. Hart, D.P. PIV error correction. Exp. Fluids 2000, 29, 13-22. [CrossRef]

20. Westerweel, J. Theoretical analysis of the measurement precision in particle image velocimetry. Exp. Fluids 2000, 29, S003-S012. [CrossRef]

21. Hsu, T.Y. Turbulent Secondary Flow in the Mixed Boundary Corner Formed by a Horizontal Free Surface and a Vertical Solid Wall. Ph.D. Thesis, Rutgers University, New Brunswick, NJ, USA, 2000.

22. Hsu, T.Y.; Grega, L.M.; Wei, T.; Leighton, R.I. Turbulent kinetic energy transport in a corner formed by a solid wall and a free surface. J. Fluid Mech. 2000, 410, 343-366. [CrossRef]

23. Legac, P.; Wei, T.; Fish, F.; Williams, T.; Mark, R.; Hutchison, S. Digital particle image velocimetry of mammalian swimming. Phys. Fluids 2008, 20, 091105. [CrossRef]

24. Stamhuis, E.J.; Nauwalaerts, S. Propulsive force calculations in swimming frogs. II. Application of a vortex ring model to DPIV data. J. Exp. Biol. 2005, 208, 1445-1451. [CrossRef] [PubMed]

25. Fish, F.E.; Beneski, J.T.; Ketten, D.R. Examination of the three-dimensional geometry of cetacean flukes using CT-scans: Hydrodynamic implications. Anat. Rec. 2007, 290, 614-623. [CrossRef] [PubMed]

26. Dearolf, J.L.; McLellan, W.A.; Dillaman, R.M.; Frierson, D., Jr.; Pabst, D.A. Precocial development of axial locomotor muscle in bottlenose dolphins. J. Morph. 2000, 244, 203-215. [CrossRef]

27. Weis-Fogh, T.; Alexander, R.M. The sustained power output from striated muscle. In Scale Effects in Animal Locomotion; Pedley, T.J., Ed.; Academic Press: London, UK, 1977; pp. 511-525.

28. Josephson, R.K. Contraction dynamics and power output of skeletal muscle. Annu. Rev. Physiol. 1993, 55, 527-546. [CrossRef] [PubMed]

29. Triantafyllou, M.S.; Triantafyllou, G.S. An efficient swimming machine. Sci. Am. 1995, 272, 40-48. [CrossRef]

30. Fish, F.E.; Hui, C.A. Dolphin swimming: A review. Mamm. Rev. 1991, 21, 181-196. [CrossRef]

31. Fish, F.E.; Rohr, J. Review of Dolphin Hydrodynamics and Swimming Performance; SPAWAR Systems Center Pacific: San Diego, CA, USA, 1999.

32. Fish, F.E. Power output and propulsive efficiency of swimming bottlenose dolphins (Tursiops truncatus). J. Exp. Biol. 1993, 185, 179-193.

33. Fish, F.E. Comparative kinematics and hydrodynamics of odontocete cetaceans: Morphological and ecological correlates with swimming performance. J. Exp. Biol. 1998, 201, 2867-2877. [PubMed]

34. Gray, J. Studies in animal locomotion VI. The propulsive powers of the dolphin. J. Exp. Biol. 1936, 13, $192-199$.

35. Fish, F.E. The myth and reality of Gray's paradox: Implication of dolphin drag reduction for technology. Bioinspir. Biomim. 2006, 1, R17-R25.

36. Flammang, B.E.; Lauder, G.V.; Troolin, D.R.; Strand, T.E. Volumetric imaging of fish locomotion. Biol. Lett. 2011, 7, 695-698. [CrossRef] [PubMed]

37. Flammang, B.E.; Lauder, G.V.; Troolin, D.R.; Strand, T. Volumetric imaging of shark tail hydrodynamics reveals a three-dimensional dual-ring vortex wake structure. Proc. Roy. Soc. B 2011, 278, 3670-3678. [CrossRef] [PubMed] 
38. Bartol, I.K.; Krueger, P.S.; Jastrebsky, R.A.; Williams, S.; Thompson, J.T. Volumetric flow imaging reveals the importance of vortex ring formation in squid swimming tail-first and arm-first. J. Exp. Biol. 2016, 219, 392-403. [CrossRef] [PubMed]

39. Skrovan, R.C.; Williams, T.M.; Berry, P.S.; Moore, P.W.; Davis, R.W. The diving physiology of bottlenose dolphins (Tursiops truncatus) II. Biomechanics and changes in buoyancy at depth. J. Exp. Biol. 1990, 202, 2749-2761.

40. Alexander, R.M. Principles of Animal Locomotion; Princeton University Press: Princeton, NJ, USA, 2003.

41. Norberg, U.M. Vertebrate Flight: Mechanics, Physiology, Morphology, Ecology and Evolution; Springer: Berlin, Germany, 1990.

42. Alexander, D.E. Nature's Flyers: Birds, Insects, and the Biomechanics of Flight; Johns Hopkins University Press: Baltimore, MD, USA, 2002.

43. Song, J.; Luo, H.; Hedrick, T. Three-dimensional flow and lift characteristics of a hovering ruby-throated hummingbird. J. R. Soc. Interface 2014, 11, 20140541. [CrossRef] [PubMed]

44. Warrick, D.R.; Tobalske, B.W.; Powers, D.R. Lift production in the hovering hummingbird. Proc. R. Soc. $B$ 2009, 276, 3747-3752. [CrossRef] [PubMed]

45. Wolf, M.; Ortega-Jimenez, V.M.; Dudley, R. Structure of the vortex wake in hovering Anna's hummingbird (Calypte anna). Proc. R. Soc. B 2013, 280, 20132391. [CrossRef] [PubMed]

46. Pournazeri, S.; Segre, P.S.; Princevac, M.; Altshuler, D.L. Hummingbirds generate bilateral vortex loops during hovering: Evidence from flow visualization. Exp. Fluids 2013, 54, 1439. [CrossRef]

47. Izrelevitz, J.S.; Triantafyllou, M.S. Adding in-line motion and model-based optimization offers exceptional force control authority in flapping foils. J. Fluid Mech. 2014, 742, 5-34. [CrossRef]

(C) 2018 by the authors. Licensee MDPI, Basel, Switzerland. This article is an open access article distributed under the terms and conditions of the Creative Commons Attribution (CC BY) license (http:/ / creativecommons.org/licenses/by/4.0/). 